\title{
Peptide substrate identification for yeast Hsp40 Ydj1 by screening the phage display library
}

\author{
Jingzhi Li and Bingdong Sha ${ }^{1 *}$ \\ 'Department of Cel Biology, Center for Biophysical Sciences and Engineering, University of Alabama at \\ Birmingham.
}

*To whom correspondence should be addressed: Bingdong Sha, Department of Cell Biology, MCLM 364, 1918 Univ. Blvd., University of Alabama at Birmingham, Birmingham, AL 35294-0005, USA. Phone: 205934-6446; Fax: 205-975-5648; Email: bdsha@uab.edu.

Submitted: May 3, 2004; Revised: September 1, 2004; Accepted: September 1, 2004; Published: October 1, 2004.

Indexing terms: Molecular chaperones; Protein folding; Heat-shock proteins 70; Peptide library.

\begin{abstract}
We have identified a peptide substrate for molecular chaperone Hsp40 Ydj1 by utilizing the combination of phage display library screening and isothemol titration calirimetry (ITC). The initial peptide substrate screening for Hsp40 Ydj1 has been carried out by utilizing a 7-mer phage display library. The peptide sequences from the bio-panning were synthesized and object to the direct affinity measurement for Hsp40 Ydj1 by isothemol titration calirimetry studies. The peptide which has the measurable affinity with Ydj1 shows enriched hydrophobic residues in the middle of the substrate fragment. The peptide substrate specificity for molecular chaperone Hsp40 has been analyzed.
\end{abstract}

\section{INTRODUCTION}

The molecular chaperones cover a large group of proteins that can recognize, bind and stabilize non-native polypeptides and facilitate protein folding (1-3). Molecular chaperone Heat shock protein 40s (Hsp40s) play critical roles in cell physiology by acting together with molecular chaperone Hsp70 members to promote protein folding, assembly, translocation, degradation and prion propagation (3). Hsp40 proteins can interact with the hydrophobic side chains of non-native polypeptides through the peptide-binding fragment and prevent the polypeptides from aggregating $(4,5)$. Hsp40s can then form the transient complexes with Hsp70s and present the non-native polypeptides to Hsp70s for subsequent protein folding (6-8). However, the mechanism by which Hsp40s recognize and bind with peptide substrate is not still elusive.
All Hsp40 proteins contain an N-terminal J-domain that can stimulate the ATPase activities of $\operatorname{Hsp} 70(1,9)$. Both type I and type II Hsp40s have a peptide-binding fragment located at the $\mathrm{C}$ terminus of the proteins (10). However, type I Hsp40 such as E. coli DnaJ, yeast Ydj1 and human Hdj2 contain two Zinc-fingerlike motifs between the J-domain and the C-terminal peptidebinding fragment within their primary sequences while type II Hsp40 proteins such as yeast Sis1 and human Hdj1 do not have these motifs $(10,11)$. It has been suggested that the Zinc-finger motifs are involved in the peptide binding for Hsp40 chaperone activities (12). It has been reported that the ability to bind nonnative polypeptides for the cytosolic Hsp40 is an essential function in vivo (13).

Molecular chaperones tend to recognize and bind peptide substrates by the hydrophobic interactions. The crystal structures

(C) 2004 by the author(s). This paper is Open Access and is published in Biological Procedures Online under license from the author(s). Copying, printing, redistribution and storage permitted. Journal (C) 1997-2004 Biological Procedures Online. 
of the members of the two major molecular chaperone families of Hsp70 and Hsp60 have been determined in complex with the peptide substrates (14-16). In the crystal structure of the peptidebinding domain of Escherichia coli DnaK (the major Hsp70 in E. coli), The Hsp70 bind the 7-mer peptide substrate in the extended conformation by internal hydrophobic residues using the peptide-binding groove. The crystal structure of the E. coli Hsp60 GroEL complexed with the peptide suggested that GroEL interacts with the peptide substrate using the hydrophobic regions at the opening of the central cavity (16).

The crystal structure of the peptide-binding fragment of Sis1, a type II yeast Hsp40 protein, has been determined in our laboratory (17). The crystal structure revealed that the Sis1 functioned as a homo-dimer with a U-shaped molecule structure. A hydrophobic depression was located on the molecular surface of the domain I of Sis1 peptide-binding fragment. We hypothesized that yeast type II Hsp40 Sis1 dimer may interact with the non-native polypeptides through the hydrophobic depressions.

The peptide-binding specificity of molecular chaperone Hsp40s and the mechanisms by which Hsp40s interact with non-native polypeptides are currently unknown. Phage display library screening has been shown to be a powerful tool for characterizing protein-peptide interactions. In this paper, we have successfully identified a peptide substrate for Hsp40 Ydj1 by screening a 7-mer phage display library. The binding affinity between the Hsp40 and the peptide substrate is measured by ITC studies. The identification of the peptide substrate has led to the structure determination of the protein complex of Hsp40 Ydj1 and the peptide substrate (18).

\section{MATERIALS AND METHODS}

\section{Protein expression and purification}

The Ydj1 peptide-binding fragment has been determined to be within amino-acid residues 102-384 of the full-length Ydj1 by limited proteolysis (10). The DNA fragment encoding Ydj1 peptide binding domain (102-384) was amplified from Ydj1 cDNA by PCR. The PCR products were digested using the restriction endonucleases $N d e \mathrm{I}$ and XhoI (New England BioLabs). The digested inserts were then ligated into the digested pet $28 \mathrm{~b}$ vector by $\mathrm{T} 4$ ligase.

$10 \mathrm{ml}$ of LB medium containing $30 \mu \mathrm{g} / \mathrm{ml}$ kanamycin was inoculated using the transformed $E$. coli stock. The cells were allowed to grow at $310 \mathrm{~K}$ in a shaker for $12 \mathrm{~h}$ and the $10 \mathrm{ml}$ of LB medium was then used to inoculate a further $1 \mathrm{~L}$ of LB medium containing $30 \mu \mathrm{g} / \mathrm{ml}$ kanamycin. $0.5 \mathrm{ml}$ of $1 M$ IPTG was added to the $1 \mathrm{~L}$ of medium in order to induce protein expression when the $\mathrm{OD}_{600}$ of the medium reached 0.6. Because Ydj1 contains Zinc finger motifs, $1 \mathrm{mM} \mathrm{ZnAc}$ was added in the culture.
The cells were harvested $3 \mathrm{~h}$ after induction. The E. coli cells from the $1 \mathrm{~L}$ of medium were pelleted down by centrifugation and resuspended in $100 \mathrm{ml}$ of $100 \mathrm{mM}$ Tris buffer $\mathrm{pH} 7.9,0.5 \mathrm{M}$ $\mathrm{NaCl}$. The cells were lysed by sonication at $277 \mathrm{~K}$. The debris and insoluble materials were pelleted down at $15,000 \mathrm{rev} / \mathrm{min}$ using a Beckman JA20 rotor. The supernatant was pumped through an Ni-charged column containing about $10 \mathrm{ml}$ resin. The column was thoroughly washed with $50 \mathrm{mM}$ Tris buffer $\mathrm{pH}$ 7.9, $0.5 \mathrm{M} \mathrm{NaCl}$ and $50 \mathrm{mM}$ imidazole in order to remove contaminating proteins. The bound protein was then eluted with $50 \mathrm{mM}$ Tris buffer $\mathrm{pH} 7.9,0.5 \mathrm{M} \mathrm{NaCl}$ and $200 \mathrm{mM}$ imidazole. The eluted protein was dialyzed against $2 \mathrm{~L}$ of $10 \mathrm{mM}$ HEPES buffer $\mathrm{pH} 7.2,150 \mathrm{mM} \mathrm{NaCl}$. The typical yield of soluble Ydj1 $(\sim 95 \%$ pure from SDS-PAGE analysis) from $1 \mathrm{~L}$ of culture is $\sim 15 \mathrm{mg}$. The N-terminal histidine tag of Ydj1 was then digested by thrombin treatment. 1 unit of thrombin (Sigma) was utilized per milligram of Ydj1 protein. Digestion took place for $12 \mathrm{~h}$ at room temperature and was stopped by the addition of $0.2 \mathrm{mM}$ PMSF. The protein was further purified on a Superdex 200 gelfiltration column (Pharmacia) mounted on an AKTA HPLC system (Pharmacia) in order to remove the thrombin and digested peptides.

\section{Phage peptide display library screening}

The Ph.D. 7-mer phage display library kit was purchased from New England Biolabs. The full-length Ydj1 was coated on the sterile polystyrene petri dish and incubated with $10 \mu$ original phage library in $1 \mathrm{ml}$ of TBS buffer (Tris $50 \mathrm{mM}$ (pH 7.5), $\mathrm{NaCl}$ $150 \mathrm{mM}$ ) with $0.1 \%$ Tween-20 for overnight at $4^{\circ} \mathrm{C}$. The dish was then extensively washed ten times with TBS buffer with $0.1 \%$ Tween-20 to minimize the nonspecific interactions between Ydj1 and the peptides expressed on phage surfaces. The bound phages were eluted by $1 \mathrm{ml}$ TBS buffer with $0.2 \mathrm{mg} / \mathrm{ml}$ Ydj1 protein after $1 \mathrm{hr}$ incubation at room temperature. The eluted phages were amplified by infecting E. coli host strain ER2738. In the next three rounds of panning, approximately $2 \times 10^{11} \mathrm{pfu}$ were put in the incubation with Ydj1. The Tween-20 concentration in the washing buffer was raised to $0.5 \%$. After the fourth panning, 20 phages were randomly selected and their DNA encoding the peptide substrate was sequenced.

\section{Isothemol titration calirimetry (ITC) studies}

Measurement of binding between Ydj1 and peptide substrates was carried out by use of an isothermal titration calorimeter (MicroCal) at room temperature. Ydj1 (or the mutant) and peptides were dialyzed against the same buffer $(10 \mathrm{mM} \mathrm{2-(4-}$ morpholino)-Ethane Sulfonic Acid) (pH 6.0), $100 \mathrm{mM} \mathrm{NaCl}, 1$ $\mathrm{mM} 2$-mercaptoethanol). Ydj1 (or the mutant) was filled in the calorimetric cell and the synthetic peptide was injected into the cell by a $250 \mu$ injection syringe. The released heat was obtained by integrating the calorimetric output curves. Pure buffers were injected into the Ydj1 protein as control experiments. The heat releases from the control experiments were subtracted from the experimental data before the data were utilized for $K_{d}$ fitting. The 
$K_{\mathrm{d}}$ values and the binding ratios were calculated by the software supplied with the calorimeter.

\section{Luciferase refolding assay}

Luciferase $(15 \mathrm{mg} / \mathrm{ml}$, Promega) was diluted by 42 times into the denaturant solution (30 mM Hepes ( $\mathrm{pH}$ 7.5), $50 \mathrm{mM} \mathrm{KCl}, 10$ $\mathrm{mM} \mathrm{MgCl}, 7 \mathrm{M}$ urea) and incubated at room temperature for 40 minutes. Then the denatured luciferase was diluted 125 times into the refolding solution $125 \mu \mathrm{l} 30 \mathrm{mM}$ Hepes (pH 7.5), $50 \mathrm{mM}$ $\mathrm{KCl}, 10 \mathrm{mM} \mathrm{MgCl}, 1 \mathrm{mM}$ ATP, $1.6 \mu \mathrm{M}$ Ydj1 or various mutants, $0.8 \mu \mathrm{M}$ Hsp70 Ssa1). Luciferase activity was determined by luciferase assay kit (Promega).

\section{RESULTS AND DISCUSSION}

\section{Identify Hsp40 Ydj1 peptide substrate candidates by screening the 7-mer phage peptide display library}

To screen the peptide substrates for Hsp40-Ydj1, we took advantage of a commercially available M13 phage peptide display library from New England Biolabs. The phage peptide display library screen has been shown to be a powerful tool to study protein-peptide interaction (19). The phage carry random amino acid fragment fused at the N-terminal of the pIII surface coat protein (New England Biolabs). The peptide is followed by a short sequence (GGGS) and then the wild type pIII coat protein.

The crystal structure of yeast Hsp40 Sis1 peptide-binding fragment indicated that a hydrophobic depression on domain I may function as the putative peptide substrate binding site for Hsp40 (17). This depression can only accommodate side chains of several hydrophobic residues. Therefore, a 7-mer phage library is considered proper to mimic the binding substrate for Ydj1. After four cycles of bio-panning, 20 colonies of the bound phages by Ydj1 were randomly chosen for subsequent DNA extraction and sequencing. Table 1 lists the sequences of the peptide substrate candidates of $\mathrm{Ydj} 1$ revealed by 7 -mer phage peptide library screening. In 20 selected sequences for Ydj1 peptide substrate candidates, nine sequences of them are distinct. Peptides GWLYEIS, SESDPVA, AWIEVLA and HWTELIE show the redundancy of $6,4,3$ and 2 times respectively (18).

Table 1: Peptide sequences for the binding phages identified by 7 mer phage peptide library screening. The numbers after the sequences indicate the redundancy of the peptide sequence from the screening.

$\begin{array}{cc}\text { GWLYEIS } & 6 \\ \text { SESDPVA } & 4 \\ \text { AWIEVLA } & 3 \\ \text { HWTELIE } & 2 \\ \text { YTVQLSS } & 1 \\ \text { DYRLIIP } & 1 \\ \text { SPWNNAN } & 1 \\ \text { YTVQLSS } & 1 \\ \text { KLFPVTK } & 1\end{array}$

The result of the phage peptide library screening indicates that yeast Hsp40 Ydj1 favors hydrophobic amino acid residues. The hydrophobic residues in the Ydj1 peptide substrate candidate sequences from the screening are more than $50 \%$ and tend to be in tandem. Most of the peptide substrate candidates contain a hydrophobic core about 3 to 4 residues. Our results are similar with the peptide substrates of E. coli Hsp40 DnaJ screened from 1633 peptides derived from 14 proteins (20). The hydrophobic patch may locate in the center, $\mathrm{N}$-terminus or C-terminus within the peptide. The hydrophobicity of these motifs in the Hsp40 peptide substrate sequences may explain that Hsp40 as a molecular chaperone can prevent protein aggregations due to hydrophobic interactions.

The phage display from Ph.D.-7 library gave the consensus results. The resultant peptide sequence with the most redundancy may be the most favorable ligand for Hsp40 Ydj1. There are the multiple DNA sequences encoding the same consensus peptide sequences in our results, which is consistent with the control experiments from New England Biolabs. The differences between the DNA sequences are usually within only one or two nucleotides. The reason for that probably lies in the degenerated genetic codon used in the phage peptide display library. The randomized region of the library encodes all 20 amino acid by using only 32 codons.

\section{ITC studies to identify the peptide substrate binding Ydj 1}

The binding affinity between a given peptide for Ydj1 can be measured by Isothermal Titration Calorimetry (ITC) method. These peptide substrate candidates for Hsp40 Ydj1 revealed by screening phage display library were synthesized (Invitrogen). These peptides were further purified to more than 95\% homogeneity using a C-18 reverse phase column mounted to the AKTA purifier system (Pharmacia). The ITC experiments were carried out by injecting the peptide solution into the buffer containing Ydj1. The heat release recorded for every reaction was integrated for affinity and stiochiometry calculation. The sensitivity of ITC instrument can allow us to detect the binding affinity with the $K_{d}$ up to around $150 \mu \mathrm{M}$. Among the 9 peptide substrate candidates, we identified one peptide with sequence GWLYEIS can bind with Ydj1 with significant affinity (Fig. 1, Table 2).

Table 2: The binding affinities between Ydj1 and the synthesized peptides measured by ITC. The ITC studies also generated the molar binding ratios between the peptides and the Ydj1 monomer by fitting the experimental data to the standard curve (N.D. not detectable).

$\begin{array}{ccc}\text { Peptides } & \begin{array}{c}\text { Dissociation } \\ \text { constants }(\boldsymbol{\mu M})\end{array} & \begin{array}{c}\text { Binding molar } \\ \text { ratios }\end{array} \\ \text { GWLYEIS } & 12 \pm 5.0 & 1.0 \pm 0.21 \\ \text { GWWYEIS } & 23 \pm 10.5 & 1.2 \pm 0.24 \\ \text { GWAYEIS } & 13 \pm 6.6 & 1.0 \pm 0.28 \\ \text { GWNYEIS } & \text { N.D. } & \text { N.D. } \\ \text { GWGYEIS } & \text { N.D. } & \text { N.D. } \\ \text { GWLYEI } & 31 \pm 13.7 & 0.8 \pm 0.22 \\ \text { GWLYE } & \text { N.D. } & \text { N.D. } \\ \text { GWLYEIS (D form) } & \text { N.D. } & \text { N.D. } \\ \text { SIEYLWG } & \text { N.D. } & \text { N.D. } \\ \text { GWLYRIS } & 20 \pm 8.4 & 0.9 \pm 0.19\end{array}$


The dissociation consent can reach $12 \mu \mathrm{M}$ with the enthalpy change $\Delta \mathrm{H}-6067 \pm 2270 \mathrm{cal} / \mathrm{mol}$ for this peptide. The ITC data also suggested that one Ydj1 dimer can interact with two peptide substrates (Table 2). Presumably one Ydj1 monomer may bind one peptide substrate. The other peptide candidates revealed by phage display library screening show no detectable affinity with Ydj1 by ITC method.

Time (min)

$-100102030405060708090100110120130140$

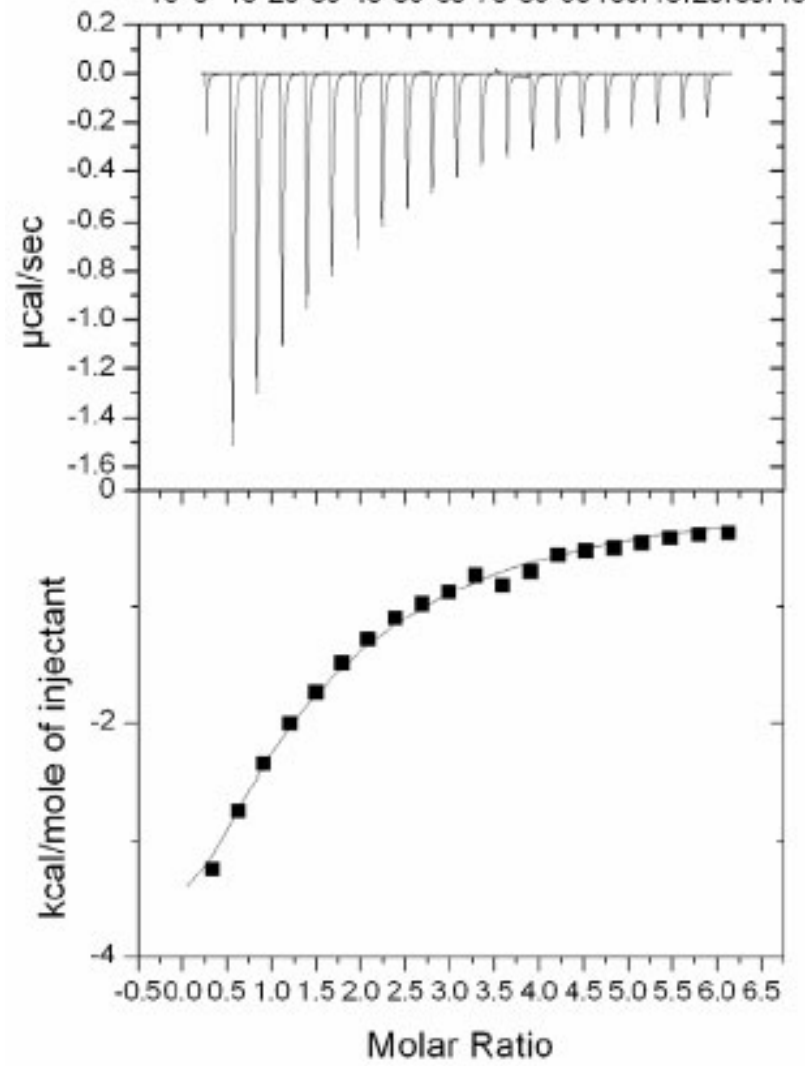

Fig. 1: ITC data of Ydj1 with the peptide GWLYEIS. The top panel shows the heat release data for injecting the buffer containing the peptide GWLYEIS in the buffer containing Ydj1. Twenty injections were performed. The lower panel shows the data fitting for the released heat from the reactions with the standard model curve.

\section{The Ydj1 peptide GWLYEIS can compete with the denatured luciferase binding site on Ydj 1}

To confirm that the identified Ydj1 peptide substrate GWLYEIS interacts with Ydj1 through the same binding site as the nonnative polypeptides do, we have tested whether the peptide GWLYEIS could compete with the denatured Luciferase in the Hsp40/Hsp70 molecular chaperone refolding machineries (Fig. 2). Purified recombinant yeast Hsp40 Ydj1 was mixed with purified yeast Hsp70 Ssa1 to constitute the in vitro Hsp40/Hsp70 system. The data from this competition assays clearly showed that the identified Ydj1 peptide substrate GWLYEIS can inhibit the refolding ability of Ydj1/Ssa1 in a dose-dependent manner (Fig. 2). At the concentration of $400 \mathrm{uM}$, peptide GWLYEIS can inhibit more than $60 \%$ of the refolding ability of Hsp40/Hsp70 system. It is highly likely that the peptide GWLYEIS inhibits the refolding ability of Ydj1/Ssa1 by competing with the denatured Luciferase to bind Ydj1 through the same sites. Therefore, by utilizing the combination of peptide display library screening, ITC studies and Luciferase competition assays, we have successfully identified a peptide substrate GWLYEIS for yeast type I Hsp40 Ydj1 (18).

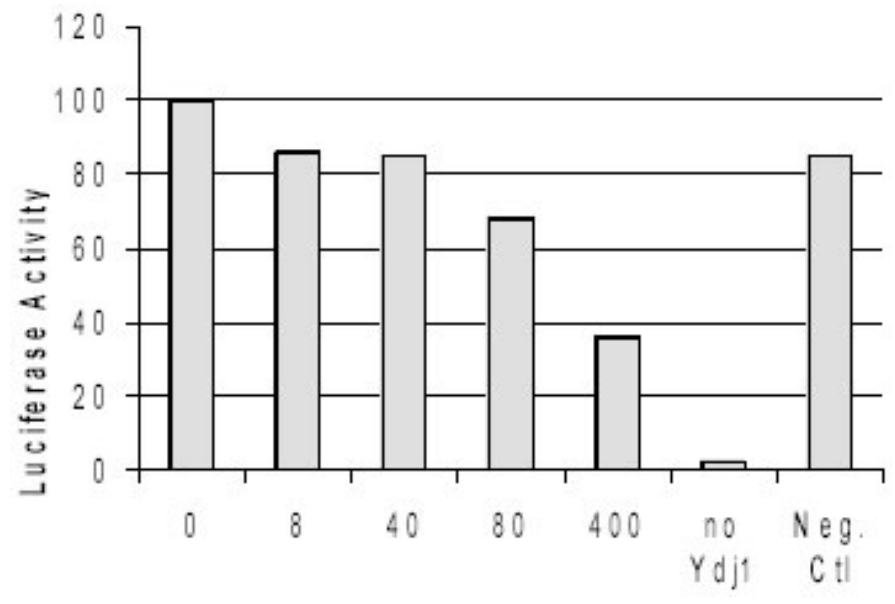

Peptide concentration ( $u M$ )

Fig. 2: The Ydj1 peptide substrate GWLYEIS competes with the denatured luciferase in Hsp40/Hsp70 molecular chaperone system. Purified recombinant Ydj1 $1.6 \mu \mathrm{M}$ was mixed with $0.8 \mu \mathrm{M}$ purified yeast Hsp70 Ssa1 in 25 $\mathrm{mM}$ Hepes buffer ( $\mathrm{pH} 7.4$ ), $50 \mathrm{mM} \mathrm{NaCl}, 5 \mathrm{mM} \mathrm{MgCl} 2$ and $1.6 \mathrm{mM}$ ATP to constitute the renaturing buffer. Various concentrations $(0 \mu \mathrm{M}, 8 \mu \mathrm{M}, 40 \mu \mathrm{M}, 80$ $\mu \mathrm{M}$ and $400 \mu \mathrm{M})$ of the Ydj1 peptide substrate were added to the buffer. Denatured Luciferase $50 \mathrm{nM}$ was diluted into the renaturing buffer and the luciferase activities were measured at 50 minute reaction. The reaction volumes are $125 \mu \mathrm{L}$. The horizontal axis indicates peptide substrate in $\mu \mathrm{M}$. The refolded luciferase activity with wild-type Ydj1 and Hsp70 Ssa1 is defined as 100\% in this Figure. As the negative control, no Ydj1 was added into the reaction buffer to generate the data curve labeled as "No Ydj1." A peptide GLYEIS with no detectable binding affinity to Ydj1 was used as negative control. At the concentration of $400 \mathrm{uM}$, the peptide GLYEIS only inhibit less than $20 \%$ of the Hsp40/Hsp70 chaperone activity and the data is labeled as "Neg. Ctl" in the figure. All data are the averaged values of three independent experiments.

\section{Characterizing the peptide substrate specificity of Hsp40 Ydj 1}

The identification of the peptide substrate of Hsp40 Ydj1 has lead to the crystal structure determination of the Ydj1-peptide complex (18). The complex crystal structure predicted that the central Leucine residue in the peptide substrate GWLYEIS may play important roles in mediating the Hsp40-peptide interactions. To investigate the peptide substrate specificity for Hsp40 Ydj1, we tried to synthesize different peptides designed on the basis of the substrate GWLYEIS (Table 2). The replacement of the Leu by a hydrophobic residue Trp or Ala within the peptide substrate GWLYEIS will not affect the binding affinity much. However, disruption of the middle hydrophobic core by substitution Leu with Gly or the polar residue Gln will decrease the binding affinity dramatically (Table 2). Substitution of the negative residue Glu within the peptide substrate GWLYEIS for a positively-charged Arg has no reduction on binding (Table 2). 
This suggests that Hsp40 may prefer a hydrophobic residue in the middle of the peptide substrate.

To identify the minimum length that is needed for the peptide substrate to bind Hsp40 Ydj1, we have synthesized peptides with the $\mathrm{N}$-terminal or the $\mathrm{C}$-terminal residues removed from the current Ydj1 peptide substrate GWLYEIS. The binding affinities between the synthesized peptides and the Hsp40 Ydj1 were measured by ITC technique (Table 2). The data suggested that the C-terminal Serine residue was dispensable for the peptide substrate binding to Ydj1. Removal of further residues from the original sequence resulted in great reduction in binding affinity between Ydj1 and the synthetic peptides. This indicates that the minimum length is required for the Hsp40 Ydj1 peptide substrate is six residues.

Type I Hsp40 DnaJ from E. Coli can bind the D-form peptide and the peptide with retro sequence with almost the same affinity or even higher $(20,21)$. However for type I Hsp40 Ydj1 from Yeast, the interactions between Ydj1 and D-form peptide substrate GWLYEIS or the peptide with the retro sequence of GWLYEIS can not be detected by ITC method (Table 2). This shows that the molecular chaperone Hsp40 may prefer certain types of peptides over others. In other words, the structure of the molecular chaperone Hsp40 may define the specificity for its peptide substrates. This phenomenon has also been shown in other molecular chaperone families (22). The peptide substrate specificities for molecular chaperones may allow them to efficiently recognize the unfolded peptide for the subsequent protein folding.

\section{ACKNOWLEDGMENTS}

We would like to thank Dr. Elizabeth Craig for providing yeast strain MW141 for Ssa1 over-expression. We would like to thank Dr Douglas Cyr for cDNA of Ydj1. The work is supported by NIH R01 (DDK56203 and R01 GM65959) and NASA to B.D.S.

\section{REFERENCES}

1. Bukau B, Horwich AL. The Hsp70 and Hsp60 chaperone machines. Cell 1998; 92:351-366.

2. Hartl FU. Molecular chaperones in cellular protein folding. Nature 1996; 381:571-580.

3. Hartl FU, Hayer-Hartl M. Molecular chaperones in the cytosol: from nascent chain to folded protein. Science 2002; 295:1852-1858.

4. Langer T, Lu C, Echols H, Flanagan J, Hayer MK, Hartl FU. Successive action of DnaK, DnaJ and GroEL along the pathway of chaperone-mediated protein folding. Nature 1992; 356:683-689.

5. Schmid D, Baici A, Gehring H, Christen P. Kinetics of molecular chaperone action. Science 1994; 263:971-973.

6. Laufen T, Mayer MP, Beisel C, Klostermeier D, Mogk A, Reinstein J, Bukau B. Mechanism of regulation of Hsp70 chaperones by DnaJ cochaperones. Proc Natl Acad Sci 1996; 96:5452-5457.

7. Misselwitz S, Staeck O, Rapoport TA. J proteins catalytically activate Hsp70 molecules to trap a wide range of peptide sequences. Mol Cell 1998; 2:593-603.

8. Qian X, Hou W, Li Z, Sha BD. Direct interactions between molecular chaperones Hsp70 and Hsp40: yeast Hsp70 Ssa1 binds the extreme C-terminal region of yeast Hsp40 Sis1. Biochem J 2002; 361:27-34.

9. Wall D, Zylicz M, Georgopoulos C. The N-terminal 108 amino acids of the E. coli DnaJ protein stimulate the ATPase activity of DnaK and are sufficient for lamda replication. $J$ Biol Chem 1994; 269:5446-5451.

10. Lu Z, Cyr DM. The conserved carboxyl terminus and zinc finger-like domain of the co-chaperone Ydj1 assist Hsp70 in protein folding. J Biol Chem 1998; 273:5970-5978.

11. Caplan AJ, Douglas MG. Characterization of Ydj1: a yeast homologue of the bacteria dnaJ protein. J Cell Biol 1991; 114:609-621.

12. Lu Z, Cyr DM. Protein folding activity of Hsp70 is modified differentially by the Hsp40 co-chaperone Sis1 and Ydj1.J Biol Chem 1998; 273:27824-27830.

13. Szabo A, Korszun R, Hartl FU, Flanagan J. A zinc fingerlike domain of the molecular chaperone DnaJ is involved in binding to denatured protein substrates. EMBO J 1996; 15:408-417.

14. Johnson JL, Craig EA. An essential role for the substratebinding region of Hsp40s in Saccharomyces cerevisiae. J Cell Biol 2001; 152:851-856.

15. Flynn GC, Pohl J, Flocco MT, Rothman JE. Peptide-binding specificity of the molecular chaperone Bip. Nature 1991; 353:726-730.

16. Zhu X, Zhao X, Burkholder WF, Gragerov A, Ogata CM, Gottesman ME, Hendrickson WA. Structural analysis of substrate binding by the molecular chaperone DnaK. Science 1996; 272:1606-1614.

17. Chen L, Sigler PB. The crystal structure of a GroEL/peptide comlex: plasticity as a basis for substrate diversity. Cell 1999; 99:757-768.

18. Sha BD, Lee S, Cyr DM. The crystal structure of the peptide-binding fragment from the yeast Hsp40 protein Sis1. Structure 2000; 8:799-807.

19. Li J, Qian X, Sha B. The crystal structure of the yeast Hsp40 Ydj1 complexed with its peptide substrate. Structure 2003; 11:1475-1483.

20. Scott JK, Smith GP. Searching for peptide ligands with an epitope library. Science 1990; 249:386-390.

21. Rudiger S, Schneider-Mergener J, Bukau B. Its substrate specificity characterizes the DnaJ co-chaperone as a scanning factor for the DnaK chaperone. EMBO J 2001; 20:1042-1050.

22. Feifel B, Schonfeld HJ, Christen P. D-peptide ligands for the co-chaperone DnaJ. J Biol Chem 1998; 273:11999-12002.

23. Siegers K, Bolter B, Schwarz JP, Bottcher UM, Guha S, Hartl FU. TRiC/CCT cooperates with different upstream chaperones in the folding of distinct protein classes. EMBO J 2003; 22: 5230-5240. 